\title{
FINITE DINILPOTENT GROUPS OF SMALL DERIVED LENGTH
}

\author{
JOHN COSSEY and YANMING WANG
}

\author{
Dedicated to Mike (M. F.) Newman on the occasion of his 65th birthday
}

(Received 16 October 1998; revised 30 August 1999)

Communicated by R. B. Howlett

\begin{abstract}
A finite dinilpotent group $G$ is one that can be written as the product of two finite nilpotent groups, $A$ and $B$ say. A finite dinilpotent group is always soluble. If $A$ is abelian and $B$ is metabelian, with $|A|$ and $|B|$ coprime, we show that a bound on the derived length given by Kazarin can be improved. We show that $G$ has derived length at most 3 unless $G$ contains a section with a well defined structure; in particular if $G$ is of odd order, $G$ has derived length at most 3 .
\end{abstract}

1991 Mathematics subject classification (Amer. Math. Soc.): primary 20D60.

Keywords and phrases: finite soluble groups, dinilpotent groups, derived length.

\section{Introduction}

If a finite group $G$ can be written as the product $A B$ of two nilpotent subgroups, $A$ and $B$, we will call $G$ a dinilpotent group. If $A$ and $B$ are of coprime order and $G$ is soluble, Hall and Higman proved that the derived length of $G$ is at most the sum of the nilpotency classes of $A$ and $B$ (as a special case of [3, Theorem 1.2.4]). Wielandt proved that a dinilpotent group $G$ must indeed be soluble if the factors are of coprime $\operatorname{order}([9])$ and Kegel then proved that a dinilpotent group is always soluble ([8]). However a bound for the derived length of dinilpotent groups has proved elusive.

When $A$ and $B$ are coprime, the bound of Hall and Higman is best possible for small values of the nilpotency classes of $A$ and $B$. However it seemed likely that for larger values of the nilpotency classes this bound is too large and should be replaced by a function of the derived lengths of $A$ and $B$. Such a bound has recently been

(C) 1999 Australian Mathematical Society 0263-6115/99 \$A2.00+0.00 
provided by Kazarin [7] in a more general setting. We denote by $d(H)$ the derived length of a soluble group $H$. For a dinilpotent group $G$ with $A$ and $B$ of coprime order he establishes that $d(G) \leq 2 d(A) d(B)+d(A)+d(B)$ and if $G$ is of odd order then $d(G) \leq d(A) d(B)+\max \{d(A), d(B)\}$ ([7, Theorem 3]) and (in the proof of [7, Corollary]) he observes that if $A$ is abelian then $d(G) \leq 2 d(B)+1$ and if further $G$ is of odd order then $d(G) \leq 2 d(B)$.

The purpose of this paper is to give more precise information about the derived length of the dinilpotent group $G$ in the case when $A$ and $B$ are of coprime orders and $A$ is abelian, $B$ metabelian. In this case, Kazarin's bounds give $G$ of derived length at most 5 and, if $G$ is of odd order, of derived length at most 4 . We will show that the bounds can be improved to 4 and 3, respectively and that these bounds are best possible. Our main result is however rather more technical and shows that in most situations the bound will be 3 and that the groups with derived length 4 have a well defined structure. In particular, we obtain that the derived length is at most 3 if $G$ has odd order.

If $A$ is abelian and $B$ is metabelian then $A$ wr $B$, the wreath product of $A$ and $B$ has derived length 3 and so the bound of 3 can not be improved. If we take $G=G L(2,3)$, then $G=A B$, where $A$ is a Sylow 3-subgroup and $B$ is a Sylow 2-subgroup. We then have $G$ of derived length $4, A$ abelian and $B$ metabelian, so that the bound of 4 for dinilpotent groups of even order can not be improved. This group is typical of the groups of derived length 4 . We say that a group $G$ is of type $(E)$ if it has the following structure: $F(G)$ is an extraspecial 2-group, $G / F(G)$ is dihedral of order $2 q$ for some odd prime $q$ and $F(G) / \Phi(F(G))$ is either a minimal normal subgroup of $G / \Phi(F(G)$ ) or the product of 2 minimal normal subgroups of $G / \Phi(F(G))$. We give examples to show that for any odd prime $q$ both these possibilities occur.

Our main result is then the following theorem.

THEOREM 1. Suppose that $G$ is a finite group and $G=A B$ with $A$ abelian and $B$ metabelian and nilpotent. Suppose further that the order of $A$ and the order of $B$ are coprime. Then $G$ is soluble of derived length at most 4 . Further, the derived length is at most 3 unless $G$ has a section of type $(E)$; in which case it has derived length 4.

\section{Preliminaries}

We begin with the observation that groups of type $(E)$ are easy to find and it is probably not difficult to classify them completely. For a given odd prime $q$ we show that we can construct groups of type $(E)$. Let $D$ denote the dihedral group of order $2 q$ and let $U$ be a faithful irreducible module for $D$ over the field of 2 elements. Then $|U|=2^{r}$, where $r$ is the order of 2 modulo $q$ if this order is even and twice the order of 2 modulo $q$ if this order is odd. It is not difficult to see that $U$ is isomorphic to 
its dual $V$ (see Doerk and Hawkes [2, Definition B.6.6] for the definition of duality). It then follows that the trivial module is a quotient of $U \otimes V$ and we can use the construction of Huppert [5, Hilfssatz 6.7.22] to give an extraspecial group $F$ of order $p^{2 r+1}$ on which $D$ acts so that $F^{\prime}$ is trivial and $F / F^{\prime} \cong U \oplus V$ as $D$-modules. Put $G=F D$. Then $G$ is clearly a group of type $(E)$. For another example, we note that there exists a non-singular $D$-invariant quadratic form on $U$ (Huppert and Blackburn [6, Theorem 7.8.13 and Theorem 7.8.30]). Thus $D$ may be regarded as a subgroup of one of the two orthogonal groups $G O_{r}^{\epsilon}(2)(\epsilon=+1$ or -1 ; see [1, page (xii)]). It then follows follows from Huppert [5, Satz 3.13.8 and Bemerkung 3.13.9(b)] that there is an extraspecial group $F$ of order $2^{r+1}$ whose automorphism group contains a subgroup $D_{0} \cong D$ for which the action of $D_{0}$ on $F / F^{\prime}$ is the same as that of $D$ on $U$. We set $G=F D$ and again $G$ is clearly a group of type $(E)$. We can vary these examples to produce non-splitting examples of a similar structure.

LEMMA 1. Let $p$ be a prime and $K$ a field of characteristic $p$. Let $G$ be a $p$ nilpotent group, $P$ a Sylow p-subgroup of $G$ and $Q=O_{p^{\prime}}(G)$ a Hall $p^{\prime}$-subgroup of $G$. Suppose that $U$ is a faithful irreducible $K G$-module. Then if $Q$ is abelian and $P$ is nonabelian, the semidirect product of $U$ and $P$ has derived length at least 3.

PROOF. Note that for $p$ an odd prime, the result is an immediate corollary of Kazarin [7, Lemma 9]. A direct proof is easy however and we include it here.

We assume that the result is false and $G$ has been chosen to have order as small as possible with $U P$ metabelian. Thus if $P_{0}$ is a nonabelian maximal subgroup of $P$ and $U_{0}$ is an irreducible submodule of $U_{Q P_{0}}$ then it is an easy consequence of Clifford's Theorem (Huppert [5, Hauptsatz V.17.3]) that $U_{0}$ and $Q P_{0} / C_{Q P_{0}}\left(U_{0}\right)$ satisfy the hypotheses of the lemma and hence $U_{0} P_{0} \leq U P$ has derived length at least 3 , a contradiction. It follows that $P_{0}$ is abelian and so every maximal subgroup of $P$ is abelian. We then have that $P$ is generated by two elements, $x$ and $y$ say, $\Phi(P)$, the Frattini subgroup of $P$, is central (and so $\Phi(P)=\zeta(P)$, the centre of $P$ ), and $P^{\prime}$ is central of order $p$. For a maximal subgroup $P_{0}$ of $P$ and an irreducible submodule $U_{0}$ of $U_{Q P_{0}}$, it is again an easy consequence of Cliffords Theorem that, if any element of $\zeta(P)$ centralises $U_{0}$, it will centralise all irreducible components of $U_{Q P_{0}}$ and hence $U$, a contradiction. Set $G_{1}=\left(Q P_{0}\right) / C_{Q P_{0}}\left(U_{0}\right)$ and let $Q_{1}$ and $P_{1}$ denote the images of $Q$ and $P_{0}$ in $G_{1}$. We now claim that $U_{0}$, regarded as a $K G_{1}$-module by deflation contains a submodule isomorphic to $K P_{1}$ when restricted to $P_{1}$. To see this note first that we may assume that $K$ is algebraically closed. Then $\left(U_{0}\right)_{Q_{1}}$ can be written as a direct sum of homogeneous components by Clifford's Theorem. Since $Q_{1}$ is abelian and $K$ is algebraically closed we have that the homogeneous components are one dimensional and moreover no element of $P_{1}$ can fix every homogeneous component. It now follows that $P_{1}$ acts faithfully and transitively as permutation group on the 
homogeneous components. But then $P_{1}$ acts regularly as permutation group on the homogeneous components (Wielandt [10, Proposition 4.4]). It is then clear that $U_{0}$ is isomorphic to $K P_{1}$ (as $K P_{1}$-module).

Suppose now that $|\zeta(P)|>2$. We have shown above that $\zeta(P) \cap C_{Q P_{0}}\left(U_{0}\right)=1$ and hence we have $\left(U_{0}\right)_{\zeta(P)}$ contains a submodule $V$ say isomorphic to $K \zeta(P)$. If now $c$ is an element of order $p$ in $P^{\prime}$ and $C=\langle c\rangle$ and $W$ is the unique maximal submodule of $V$ then $W_{C}$ contains a submodule isomorphic to $K C$ unless $C=\zeta(P)$, in which case we must have $p \geq 3$ and $W$ uniserial of length $p-1$. In either case we have that for some element $w \in W, w c \neq w$. Next suppose that $|\zeta(P)|=2$. Then $P$ contains a cyclic subgroup of order 4 ; we may assume that $P_{0}$ has been chosen to be cyclic (of order 4). In this case we have $C_{P_{0}}\left(U_{0}\right)=1$ and so $\left(U_{0}\right)_{P_{0}}$ contains a submodule $V$ isomorphic to $K P_{0}$. If $W$ is the unique maximal submodule of $V$, then $W_{\zeta(P)}$ contains a submodule isomorphic to $K \zeta(P)$. Again if $1 \neq c \in P^{\prime}$ there is an element $w \in W$ such that $w c \neq w$.

We now translate the claims above in the semidirect product $P U$. We have an element $1 \neq c \in P^{\prime}$ and $w$ in the radical of $U$ such that $w c-w \neq 0$. In the semidirect product, $w \in[U, P]$ and $w c-w$ may be written $[w, c]$. But both $w$ and $c$ are in $(U P)^{\prime}$ and so $(U P)^{\prime \prime} \neq 1$. This completes the proof of the lemma.

The next lemma generalises a result from modular representation theory in a form we need.

LEMMA 2. Let $p$ be a prime, $G$ a group with $U$ an abelian normal $p$-subgroup and $G / U$ a p-nilpotent group. Then $U=U_{1} \times \cdots \times U_{t}$ where each $U_{i}$ is normal in $G$, all chief factors of $G$ contained in $U_{i}$ are isomorphic as $G$-modules and if $i \neq j$ no chief factor of $U_{i}$ is isomorphic to a chief factor of $U_{j}$.

PROOF. When $U$ is elementary abelian, we can regard $U$ as a $G / U$-module and the result is then essentially a restatement of a theorem of Srinivasan (Huppert and Blackburn [6, Theorem 7.16.10]). We proceed by induction on the length of a $G$ chief series from $U$ to 1 ; the result is clearly true for 1 . By our observation we can assume that $U$ is not elementary abelian. If $U$ has exponent $p^{a}$ then $U^{p^{a-1}}$ is elementary abelian and moreover isomorphic (as an $G$-module) to a quotient of $U / \Phi(U)$. Since $U^{p^{a-1}} \leq \Phi(U)$, we have that for some minimal normal subgroup $V$ of $G$ contained in $U U / V$ contains a $G$-chief factor isomorphic to $V$ (as $G$ modules). Now by our inductive hypothesis $U / V$ can be written as a direct product $U / V=\left(U_{1}^{*} / V\right) \times\left(U_{1}^{*} / V\right) \times \cdots \times\left(U_{t}^{*} / V\right)$, where the $U_{j}^{*} / V$ satisfy the requirements of the lemma and $U_{1}^{*} / V$ has been chosen so that each chief factor of $G$ contained in $U_{1}^{*} / V$ is isomorphic to $V$. For $i>1$ we have the length of $U_{i}^{*}$ is less than the length of $U$ and so $U_{i}^{*}=V \times U_{i}$, since no chief factor of $U_{i}^{*} / V$ is isomorphic to $V$. Set 
$U_{1}=U_{1}^{*}$. Then it is easy to see that $U=U_{1} \times \cdots \times U_{t}$ and that the $U_{i}$ satisfy the requirements of the lemma.

The next result is a technical one we need in the proof of the main theorem.

LEMMA 3. Let $p, q$ be distinct primes and suppose $G=A B$, where $A$ is the unique minimal normal subgroup of $G$ and is of q-power order and $B$ is cyclic of order $p^{i}$. Let $U$ be a faithful irreducible $F G$-module, where $F$ is a finite field of characteristic $p$. Let $V$ be the radical of $U_{B}$. Then $U_{B}$ is a free $F B$-module (of rank $t$ say) and for any element $1 \neq a \in A$ we have $V+V a=U$. Further, $U /(V \cap V a)$ has dimension at most $2 t$.

PROOF. Recall that the radical of a module is the smallest submodule with completely reducible quotient (Doerk and Hawkes [2, Definition B.3.7] and remarks following). If $F$ is a splitting field for $G$, then $U$ is induced from a 1-dimensional irreducible for $A$ (by Clifford's Theorem) and so by the Mackey Subgroup Theorem (Huppert [5, Satz V.16.9]) $U_{B}$ is a free $F B$-module. It then follows easily that $U_{B}$ is free for any field $F$ of characteristic $p$. If the dimension of $U$ over $F$ is $t$, then $t=p^{i} r$ and $U_{B}$ is free of rank $r$. Note that if $a \in A$, then $V a$ is the radical of $U_{B^{a}}$. There are now two cases to consider.

Suppose first that $U_{A}$ is reducible, so that $U_{A}=U_{0} \oplus \cdots \oplus U_{p^{s}-1}$, where $s \leq t$ and each $U_{j}$ is a distinct irreducible $F A$-module of dimension $p^{t-s} r$. If $B=\langle b\rangle$, then $B$ permutes the $U_{j}$, say $U_{0} b^{j}=U_{j}$, with $0 \leq j \leq p^{s}-1$. We then have that $Y=\left\{u-u b: u \in U_{0}\right\}$ is a subspace of $U$ contained in $V$. Now suppose $1 \neq a \in A$. Then $[b, a] \neq 1$ and so $[b, a]$ does not act trivially on some $U_{j}$; we may suppose that $U_{0}$ has been chosen so that $[b, a]$ does not act trivially on $U_{0}$. Let $W=\left\{u-u b^{a}: u \in U_{0}\right\}$. Suppose now that $W \cap V \neq 0$. Since $U=U_{0} \oplus \cdots \oplus U_{p-1}$, we have $u-u b^{a}=x+y$, with $x \in U_{0}$ and $y \in U_{1}$. Since $U_{0} b^{a}=U_{1}$ we then have $x=u$ and since $x-x b \in V$ we also have $y+x b \in U_{1} \cap V=0$, giving $y=-x b$. Thus we now have $u b^{a}=u b$ and so $u[b, a]=u$. But then $[b, a]$ acts trivially on $U_{0}$, contradicting the choice of $a$. We thus have $W \cap V=0$. Since $W$ has dimension $r$ and $V$ has dimension $(p-1) r$, we have $W+V$ has dimension $p r$ and so $U=W+V$. Then we have $U=V+V a$ since $W \leq V a$.

Next we suppose that $U_{A}$ is irreducible. It follows that $A$ is cyclic of order $q$ and $p \mid q-1$. Let $E$ be the field of order $|F|^{p r}$. Then we can regard $U$ as the additive group of $E, A$ as a subgroup of the multiplicative group of $E$ and $B$ as a subgroup of the Galois group of $E$ over $F$. Note that $q$ divides $|F|^{p r}-1$ but not $|F|^{s}-1$ for any $s<p r$. If $D$ denotes the subfield of $E$ fixed element-wise by $B$, then $E$ has dimension $p$ as a vector space over $D$. We now regard $E$ as a $D G$-module and we then have $E_{B}$ is isomorphic to $D B$ as $D B$-module. The radical $W$ of $E_{B}$ then has dimension $p-1$ (over $D$ ). Since the radical of $E_{B^{a}}$ is $W a$ for any $a \in A$ and $W a \neq W$ 
if $a \neq 1$ (otherwise $W$ would be $G$-invariant, a contradiction) we have $W+W a=E$. Since $W$ regarded as an $F B$-module has dimension $r(p-1)$ and $E / W$ is trivial as $F B$-module, $W$ is the radical of $E$ as $F B$-module. But $U$ is isomorphic to $E$ as $F G$-module and the result follows.

The final statement of Lemma 3 comes immediately from the fact that the free $F B$-module of rank $t$ modulo its radical has dimension $t$.

\section{Proof of Theorem 1}

We suppose that $G$ satisfies the following hypothesis:

(*) $G=A B$ with $A$ abelian, $B$ metabelian and nilpotent and $A$ and $B$ of coprime orders. Further, $G$ has no section isomorphic to one of the groups $P(p, i)$.

We want to show that if $G$ satisfies $\left({ }^{*}\right)$ then $G$ has derived length at most 3 . So we suppose that $G$ has been chosen to have order as small as possible with derived length greater than 3 and satisfying $\left({ }^{*}\right)$. We begin with some standard reductions.

Since any quotient of a group satisfying $\left({ }^{*}\right)$ also satisfies $\left({ }^{*}\right)$, it follows quickly that $G$ has a unique minimal normal subgroup $N$ whose quotient $G / N$ has derived length 3 . We also have that $F(G)$ is a $p$-group for some prime $p$. Further if $\pi(A)$ is the set of primes dividing $|A|$, then $G$ has $\pi(A)$-length 1. If $p \in \pi(A)$ then $A$ centralises $F(G)$ and so is contained in $F(G)$ (Huppert [5, Satz 3.4.2]). Thus $A=F(G)$, $G / F(G) \cong B$ and $G$ clearly has derived length at most 3 , a contradiction. Hence we must have $p \in \pi(B)$. If $H$ is the Hall $p^{\prime}$-subgroup of $B$ then centralises $F(G)$ and so $H \leq F(G)$, giving $H=1$. Thus $B$ is a $p$-group. If $B=F(G)$ then $G / B \cong A$ and again $G$ has derived length at most 3, a contradiction.

We now have $M=F(G) A$ a normal subgroup of $G$ with $G / M$ a nontrivial $p$ group. We suppose first that $G / M$ is nonabelian, so that there are elements $x$ and $y$ in $B$ with $[x, y]$ not in $M$. It then follows from Huppert [5, Satz 3.4.2] that there is a chief factor $F(G) / K$ of $G$ with $[x, y] \notin C_{G}(F(G) / K)$. Let $H / K$ be a complement for $F(G) / K$ in $G / K$. Then the semidirect product $(F(G) / K)\left(H / C_{H}(F(G) / K)\right)$ $\cong G / C_{H}(F(G) / K)$ satisfies the hypotheses of Lemma 1 and so has Sylow $p$-subgroup of derived length at least 3 , a contradiction. Thus we must have $G / M$ abelian. Note that this immediately gives a bound of 4 for the derived length, since $G / M$ and $M / F(G)$ are abelian and $F(G)$ is metabelian. Our aim now is to show that $F(G)$ must be abelian unless $G$ has a section isomorphic to some $P_{i}$.

Since we have assumed that $G$ has derived length greater than 3 and is minimal, we have $N=G^{\prime \prime \prime}<G^{\prime \prime} \leq F(G)$. Let $L$ denote the smallest normal subgroup of $G$ contained in $F(G)$ for which every chief factor $X / Y$ of $G$ with $L \leq X<Y \leq F(G)$ satisfies $G / C_{G}(X / Y)$ abelian. Note that $L \leq G^{\prime \prime}$. Also we have that $G / L$ is nilpotentby-abelian and so since any nilpotent subgroup of $G$ is metabelian we have $G / L$ of 
derived length at most 3 . In particular $1 \neq L$ and so $N \leq L$. Since $G^{\prime \prime}$ is not abelian and $G^{\prime \prime \prime}=N$ we have $N \leq \zeta\left(G^{\prime \prime}\right)$ and so $G^{\prime \prime}$ has nilpotency class 2 . Suppose that $F(G) \neq L$.

Suppose that $L$ is abelian and let $D$ be a maximal abelian normal subgroup containing $L$. Then $D \leq F(G)$ and we must have $F(G) / D$ nonabelian. Let $E / D=(F(G) / D)^{\prime}$ and suppose that $L$ is not contained in $\zeta(E)$. We choose $L / K$ to be a chief factor of $G$ with $\zeta(E) \cap L \leq K$. If $x$ is a $p$-power element not in $F(G)$ we have $L / K$ as an $\langle x\rangle$-module is nontrivial and so for some element $y K \in L / K$ we have $[x, y] \notin K$. If $c \in F(G)^{\prime}$, then if $P$ is a Sylow $p$-subgroup of $G$ containing $x, c$ and $[x, y]$ are both in $P^{\prime}$. Thus $[c,[x, y]]=1$. Since $E$ is generated by $F(G)^{\prime}$ and $D$ we have $[x, y] \in \zeta(E)$, a contradiction. It follows that $L$ is not abelian.

Now let $F(G) / K$ be a chief factor with $L \leq K$. Then $F(G) / K$ is complemented in $G$, by $H$ say. We then have that $H$ satisfies $\left(^{*}\right)$. Moreover $L \leq H^{\prime \prime}$, since if not there is a chief factor $L / J$ of $G$ with $L$ not contained in $H^{\prime \prime} J$. But then $L / J$ is a chief factor of $H$ with $H^{\prime \prime} \cap L \leq J$ and $H / C_{H}(L / J)$ abelian. But then we have $G / C_{G}(L / J)$ abelian, a contradiction. It follows that $L \leq H^{\prime \prime}$ and then $H$ has derived length 4 , a contradiction. Thus we must have $F(G)=L$.

Since $L \leq G^{\prime \prime} \leq F(G)$ we have $G^{\prime \prime}=F(G)$. Then we have $N=G^{\prime \prime \prime} \leq \zeta(F(G))$ and so $F(G)$ is of nilpotency class 2. Moreover, since $F(G)^{\prime}$ is elementary abelian, $p^{\text {th }}$ powers are central in $F(G)$, giving $\Phi(F(G))$ central in $F(G)$. We have $G / F(G)$ $p$-nilpotent and so by Lemma 2 we can write $F(G) / N=\left(U_{1} / N\right) \times \cdots \times\left(U_{n} / N\right)$, where all chief factors between $U_{i}$ and $N$ are isomorphic and if $i \neq j$ no chief factor between $U_{i}$ and $N$ is isomorphic to a chief factor between $U_{j}$ and $N$. Note now that no chief factor $F(G) / K$ can have $G / C_{G}(F(G) / K)$ nilpotent, for we would then have $G / C_{G}(F(G) / K)$ abelian. It follows that $F(G) / N$ is the metanilpotent residual of $G / N$ and so is complemented, by $H / N$ say (Huppert [5, Satz 6.7.15]). If $F(G) / K$ is a chief factor of $G$ we put $E=K H$. If $K$ is nonabelian then $E$ satisfies $\left({ }^{*}\right)$ and has derived length 4 , a contradiction. Hence $K$ must be abelian. Suppose that $F(G) / \Phi(G)=\left(V_{1} / \Phi(G)\right) \times \cdots \times\left(V_{m} / \Phi(G)\right)$ with $V_{i} / \Phi(G)$ a chief factor of $G$. If $m>2$ then the product of any $m-1$ of the $V_{i}$ is abelian and so in particular $\left[V_{i}, V_{j}\right]=1$ and then since $F(G)$ is generated by the $V_{i}$ we have $F(G)$ abelian, a contradiction. Thus $F(G) / \Phi(G)$ is either a minimal normal subgroup or the product of two minimal normal subgroups of $G / \Phi(G)$.

We now consider the structure of $G / F(G)$. We have $B$ a Sylow $p$-subgroup of $G$ and we let $K$ be a maximal subgroup of $B$ containing $F(G)$. Then $K A$ is a normal subgroup of index $p$ in $G$ and also satisfies $\left({ }^{*}\right)$. It follows that $K A$ must have derived length 3 and hence that $(K A)^{\prime \prime}$ must be properly contained in $F(G)$. Regarded as a $\mathbb{Z}_{p}(K A)$-module, $F(G) / \Phi(G)$ is completely reducible by Clifford's Theorem and so if $F(G) / L$ is a chief factor of $G$ with $(K A)^{\prime \prime} \leq L$ we have that $K A$ acts on each composition factor of $F(G) / L$ as an abelian group. It follows that $K$ 
centralises $F(G) / L$. If $F(G) / \Phi(G)$ is irreducible or the direct sum of two isomorphic irreducibles, then we must have $K \leq F(G)$ and hence $K=F(G)$. Now suppose that $F(G) / \Phi(G)=(U / \Phi(G)) \times(V / \Phi(G))$, with $U / \Phi(G), V / \Phi(G)$ irreducible. If $B$ has two distinct maximal subgroups containing $F(G)$, it has at least $p+1$ maximal subgroups containing $F(G)$. Thus we can find distinct maximal subgroups $K_{1}, K_{2}, K_{3}$ each containing $F(G)$. We can not have both $K_{1}$ and $K_{2}$ centralising $U / \Phi(G)$, for then we would have $B$ centralising $U / \Phi(G)$ and $G$ acting on $U / \Phi(G)$ as an abelian group, a contradiction; suppose $K_{1}$ centralises $U / \Phi(G)$. On the other hand, $K_{3}$ must centralise one of $U / \Phi(G), V / \Phi(G), U / \Phi(G)$ say. We then have $B$ centralises $U / \Phi(G)$, a contradiction. Thus we may assume that $B$ has a unique maximal subgroup containing $F(G)$. It nows follows that $B / F(G)$ is cyclic and moreover that $B / F(G)$ acts faithfully on one of $U / \Phi(G)$ and $V / \Phi(G), U / \Phi(G)$ say, and then $(B / F(G))^{p}$ centralises $V / \Phi(G)$.

Note that $F(G) A$ is normal in $G$; we choose $F(G) A_{0}$ normal in $G$ and so that $(F(G) A) /\left(F(G) A_{0}\right)$ is a chief factor. We then have $B A_{0}$ satisfies $\left(^{*}\right)$ and so has derived length at most 3 . Thus we must have that $B A_{0}$ acts as abelian group on some chief factor $F(G) / W$. Since $B A_{0}$ cannot act as an abelian group on $F(G) / W$, we must have $B$ centralises $\left(F(G) A_{0}\right) / F(G)$ but not $(F(G) A) /\left(F(G) A_{0}\right)$. It now follows from Higman's Lemma [3] that $A=A_{0} \times A_{1}$ with $\left(F(G) A_{1}\right) / F(G)$ a chief factor of $G$. If $F(G) / \Phi(G)$ is irreducible, we have $B A_{1}$ of derived length 4 and so $G=B A_{1}$, giving $A=A_{1}$. Hence suppose that $F(G) / \Phi(G)$ is reducible, so that $F(G) / \Phi(G)=(U / \Phi(G)) \times(V / \Phi(H))$, with $U / \Phi(G)$ and $V / \Phi(G)$ chief factors of $G$. If $A_{1}$ does not centralise either of $U / \Phi(G)$ and $V / \Phi(G)$ then again $B A_{1}$ has derived length 4 and $A_{1}=A$. If $A_{1}$ centralises $U / \Phi(G)$ then it cannot centralise $V / \Phi(G)$ also. Moreover we must have $A_{0}$ centralises $V / \Phi(G)$, since it must centralise one of $U / \Phi(G)$ and $V / \Phi(G)$ and if it centralised $U / \Phi(G) A$ would centralise $U / \Phi(G)$, a contradiction. Now choose $F(G) A_{2}$ so that $\left(F(G) A_{0}\right) /\left(F(G) A_{2}\right)$ is a chief factor of $G$. We have then that $B A_{1} A_{2}$ has derived length at most 3 and so we must have $B A_{1} A_{2}$ acts as an abelian group on $U / \Phi(G)$. Since $B A_{0}$ does not act as an abelian group on $U / \Phi(G)$, we again see that $A_{0}=A_{2} \times A_{3}$. But then $B A_{1} A_{3}$ has derived length 4 and so $A_{3}=A_{1}$, giving $\left(F(G) A_{1}\right) / F(G)$ a chief factor of $G$ and $A=A_{0} \times A_{1}$. Note that if $A_{0} \cong A_{1}$ as $B$-modules then we may take a diagonal submodule $D$ and get $B D$ of derived length 4 , a contradiction. In particular if $A=A_{0} \times A_{1}$, we must have $|B / F(G)|>2$.

If $F(G) / \Phi(G)=(U / \Phi(G)) \times(V / \Phi(G))$ is the direct product of two minimal normal subgroups, then $U$ and $V$ are abelian and so $\Phi(G)=U \cap V$ is central in $F(G)$. If $F(G) / \Phi(G)$ is a minimal normal subgroup then $F(G) / \Phi(F(G))$ is indecomposable as $G / F(G)$-module. But $F(G) / \Phi(G)$ is faithful and free as $B / F(G)$-module and so by Lemma 3 it is free as $B / F(G)$-module. But then it is projective as $G / F(G)$ module (Huppert and Blackburn [6, Theorem 7.7.14]) and hence $\Phi(G)=\Phi(F(G)$ ). 
Since $F(G)^{\prime}=N$ is elementary abelian, we have $p^{t h}$ powers of elements of $F(G)$ are central in $F(G)$ and so again $\Phi(G) \leq \zeta(G)$. We also have $B^{\prime} \leq F(G)$ and hence $B^{\prime} \Phi(G)$ is an abelian normal subgroup of $F(G)$. Regarding $F(G) / \Phi(G)$ as a $B / F(G)$-module we have $B^{\prime} \Phi(G) / \Phi(G)$ generated by the elements $u^{-1} u^{b} \Phi(G)$, with $b \in B, u \in F(G)$, so that $B^{\prime} \Phi(G) / \Phi(G)$ is just the radical of $F(G) / \Phi(G)$.

At this point it is convenient to break the proof into a number of different cases. We have $F(G) A / F(G)$ can be a chief factor of $G$ or the direct product of two chief factors of $G, B / F(G)$ is cyclic and can have order either 2 or greater than 2 . These give rise to the following cases: $F(G) A / F(G)$ the product of two chief factors with $|B / F(G)|>2$ and $F(G) A / F(G)$ a chief factor with $|B / F(G)|=p>2$ or $|B / F(G)|=2$. Using the Frattini argument we can choose $b \in B$ so that $\langle b\rangle$ normalises $A$ and $G=F(G) A\langle b\rangle$.

Suppose first that $A=A_{0} \times A_{1}$ and $F(G) / \Phi(G)=\left(U_{0} / \Phi(G)\right) \times\left(U_{1} / \Phi(G)\right)$, with $\left[U_{1}, A_{0}\right] \leq \Phi(G)$ and $\left[U_{0}, A_{1}\right] \leq \Phi(G)$ and let $V_{i} / \Phi(G)$ denote the radical of $U_{i} / \Phi(G), i=0,1$. Suppose moreover that $|B / F(G)|=p^{r}$ and $\langle b\rangle / C_{\langle b\rangle}\left(A_{0}\right)$ has order greater than 2. Let $\left|U_{0} / \Phi(G)\right|=p^{p^{\prime} t}$ and $\left|U_{1} / \Phi(G)\right|=p^{p k}$. We then have $V / \Phi(G)=\left(V_{0} / \Phi(G)\right) \times\left(V_{1} / \Phi(G)\right)$ is the radical of $F(G) / \Phi(G)$. By Lemma 3 we can find elements $a_{i}$ such that $V_{i}^{a_{i}} / \Phi(G)$ is the radical of $U_{i} / \Phi(G)$ as $\langle b\rangle^{a_{i}}$-module and $U_{i} / \Phi(G)=\left(V_{i} / \Phi(G)\right)\left(V_{i}^{a_{i}} / \Phi(G), i=0,1\right.$. If $a=a_{0} a_{1}$ then $F(G)=V^{a} V$. Since $V$ and $V^{a}$ are abelian normal subgroups of $F(G)$, we have $V^{a} \cap V \leq \zeta(F(G))$. But by Lemma 3, we have $\left|F(G) /\left(V^{a} \cap V\right)\right| \leq p^{2 t+2 k}<|F(G) / \Phi(G)|$, since $2<p^{r}$. Thus $\zeta\left(F(G)>\Phi(G)\right.$ and hence must contain either $U_{0}$ or $U_{1}$. But then since both $U_{0}$ and $U_{1}$ are abelian, we must have $F(G)$ abelian, a contradiction.

We now suppose that $F(G) A / F(G)$ is a chief factor and hence $|B / F(G)|=p$. We consider the case $p$ odd. Then if $F(G) / \Phi(G)$ is an irreducible $H$-module, we let $|F(G) / \Phi(G)|=p^{p k}$. If $V / \Phi(G)$ is the radical of $F(G) / \Phi(G)$ as a $B$-module, it follows from Lemma 3 that if $1 \neq a \in A$ we have $F(G)=V^{a} V$. Moreover, $V^{a} \cap V \leq \zeta(F(G))$ since $V, V^{a}$ are abelian. From Lemma 3 we have $\mid F(G) /\left(V^{a} \cap\right.$ $V)\left|\leq p^{2 k}<\right| F(G) / \Phi(G) \mid$. But then $\zeta(F(G))=F(G)$, a contradiction. Hence we suppose that $F(G) / \Phi(G)=\left(U_{0} / \Phi(G)\right) \times\left(U_{1} / \Phi(G)\right)$ with $U_{0} / \Phi(G)$ and $U_{1} / \Phi(G)$ chief factors of $G$. We let $V_{i} / \Phi(G)$ be the radical of $U_{i} / \Phi(G)$ (considered as a $B$ module) and take $1 \neq a \in A$. As above we see from Lemma 3 that $\left(V_{0} V_{1}\right)^{a} \cap\left(V_{0} V_{1}\right)$ is central and properly contains $\Phi(G)$, giving a contradiction.

We are now left with the case $F(G) A / F(G)$ a chief factor and $|B / F(G)|=2$. We now have $G / F(G)$ dihedral of order $2 q$. Suppose first that $F(G) / \Phi(G)$ is a chief factor and let $V / \Phi(G)$ be the radical of $F(G) / \Phi(G)$ as $\langle b\rangle$-module. Again if $1 \neq a \in A$, we have $V, V^{a}$ both abelian, $V^{a} V=F(G)$ and since $p=2$ we have $V^{a} \cap V=\Phi(G)$. Thus we may choose generators $u_{1}, \ldots u_{k}, v_{1}, \ldots, v_{k}$ for $F(G)$ with $\left[u_{i}, u_{j}\right]=\left[v_{i}, v_{j}\right]=1$ for all pairs $1 \leq i, j \leq k$. Thus $F(G)^{\prime}$ is generated by the commutators $\left[u_{i}, v_{j}\right], 1 \leq i, j \leq k$. For a fixed $u_{i}$ and $x \in F(G)$ it is easy to 
check that the map $x \Phi(G) \rightarrow\left[u_{i}, x\right]$ is a $\langle b\rangle$-module homomorphism with $V / \Phi(G)$ in its kernel. Thus the image is a completely reducible $\langle b\rangle$-submodule of $F(G)^{\prime}$. It follows that $F(G)^{\prime}$ is a completely reducible $\langle b\rangle$-module. Since $F(G)^{\prime}$ is irreducible as $(G / F(G))$-module it cannot be faithful by Lemma 3 and hence it must be trivial. Thus we have $F(G)^{\prime}$ central in $G$. Now suppose that $\Phi(G) \neq F(G)^{\prime}$. Since all chief factors of $G$ in $F(G) / F(G)^{\prime}$ are noncentral by Lemma 2 and all chief factors of $G$ in $\zeta(F(G))=\Phi(G)$ are central by Lemma 2, we have a contradiction. Thus $F(G)^{\prime}=\zeta(G)=\Phi(G)=\Phi(F(G))$ and so $F(G)$ is extraspecial and $G$ is of type $(E)$, a contradiction. A similar argument applies if $F(G) / \Phi(G)$ is the direct product of two minimal normal subgroups of $G / \Phi(G)$, again leading to $G$ being of type $(E)$, a final contradiction.

We are now left with proving that if $G=A B, A$ abelian, $B$ metabelian and nilpotent and $A$ and $B$ of coprime order and $G$ has a section of type $(E)$, then $G$ has derived length 4 . It is enough to show that every group of type $(E)$ satisfies these conditions and is of derived length 4 . That a group of type $(E)$ has derived length 4 is clear. If $G$ is of type $(E)$, then we can write $G=A B$ where $A$ is a (cyclic) Sylow $q$-subgroup and $B$ is a Sylow 2-subgroup. We need to show that $B$ is metabelian to complete the proof. The proof is similar to the argument above. If $b$ is chosen so that $G=F(G) A\langle b\rangle$ we then let $V / \Phi(G)$ be the radical of $F(G) / \Phi(G)$ as $\langle b\rangle$-module. If $v \in V$ is fixed and $x \in F(G)$ then the map $x \Phi(G) \rightarrow[v, x]$ is a $\langle b\rangle$-module homomorphism from $F(G) / \Phi(G)$ to $\Phi(G)$. Since the image is completely reducible we have $V / \Phi(G)$ in the kernel, giving $[v, x]=1$ for all $x \in V$. Since this is true for any $v \in V$, we have $V$ abelian. That $B / V$ is abelian comes immediately from the definition of $V$ and hence $B$ is metabelian as required.

\section{References}

[1] J. H. Conway, R. Curtis, S. Norton, R. Parker and R. Wilson, Atlas of finite groups (Clarendon Press, Oxford, 1985).

[2] K. Doerk and T. O. Hawkes, Finite soluble groups, Expositions in Mathematics 4 (de Gruyter, Berlin, 1992).

[3] P. Hall and G. Higman, 'The $p$-length of $p$-soluble groups and reduction theorems for Burnside's problem', Proc. London Math. Soc. (3) 7 (1956), 1-42.

[4] G. Higman, 'Complementation of Abelian normal subgroups', Publ. Math. Debrecen 4 (1955-6), 455-458.

[5] B. Huppert, Endliche Gruppen (Springer, Berlin, 1967).

[6] B. Huppert and N. Blackburn, Finite groups II (Springer, Berlin, 1982).

[7] L. S. Kazarin, 'Soluble products of groups', in: Infinite Groups 94 (eds. F. de Giovanni and M. Newell) (de Gruyter, New York, 1995) pp. 111-123.

[8] O. H. Kegel, 'Produkte nilpotenter Gruppen', Arch. Math. 12 (1961), 90-93.

[9] H. Wielandt, 'Über Produkte von nilpotenten Gruppen', Illinois J. Math. 2 (1958), 611-618.

[10] - Finite permutation groups (Academic Press, New York, 1964). 
Mathematics Department

School of Mathematical Sciences

Australian National University

Canberra 0200

Australia

e-mail: john.cossey@maths.anu.edu.au
Department of Mathematics Zhongshan University of Guangzhou 510275 P. R. China e-mail: stswym@zsulink.zsu.edu.cn 